\title{
Erratum to: Decoherence of quantum parameter estimation for open Dirac particle in Garfinkle-Horowitz-Strominger dilation black hole
}

\author{
Yumeng Huang ${ }^{1}$, Kai Yan ${ }^{1}$, Yinzhong Wu${ }^{1}$, Xiang Hao ${ }^{1,2, a}$ \\ ${ }^{1}$ School of Mathematics and Physics, Suzhou University of Science and Technology, Suzhou 215009, Jiangsu, People's Republic of China \\ ${ }^{2}$ Pacific Institute of Theoretical Physics, Department of Physics and Astronomy, University of British Columbia, 6224 Agriculture Rd., Vancouver, \\ BC V6T 1Z1, Canada
}

Received: 9 January 2020 / Accepted: 13 January 2020 / Published online: 4 February 2020

(C) The Author(s) 2020

Erratum to: Eur. Phys. J. C (2019) 79:974

https://doi.org/10.1140/epjc/s10052-019-7491-z

There is a small change in the reference of [1], which was reference [20] in the original article. The authorship of Dr. Nicolai Friis who was a co-author on an early version of the manuscript has been withdrawn before the submission to the journal due to some scientific disagreement. Therefore, the author information of the reference has been corrected.

Acknowledgements Funded by SCOAP ${ }^{3}$.

Open Access This article is distributed under the terms of the Creative Commons Attribution 4.0 International License (http://creativecomm ons.org/licenses/by/4.0/), which permits unrestricted use, distribution, and reproduction in any medium, provided you give appropriate credit to the original author(s) and the source, provide a link to the Creative Commons license, and indicate if changes were made.

Funded by $\mathrm{SCOAP}^{3}$.

\section{Reference}

1. M. Ahmadi, D.E. Bruschi, C. Sabín, G. Adesso, I. Fuentes, Sci. Rep. 4, 4996 (2014)

The original article can be found online at https://doi.org/10.1140/ epjc/s10052-019-7491-z.

a e-mail: xhao@mail.usts.edu.cn 